\title{
Humidification of Inspired Oxygen Is Increased With Pre-nasal Cannula, Compared to Intranasal Cannula
}

\author{
Dominic Dellweg MD, Markus Wenze MD, Ekkehard Hoehn, \\ Olaf Bourgund MD, and Peter Haidl MD
}

\begin{abstract}
BACKGROUND: Oxygen therapy is usually combined with a humidification device, to prevent mucosal dryness. Depending on the cannula design, oxygen can be administered pre- or intranasally (administration of oxygen in front of the nasal ostia vs cannula system inside the nasal vestibulum). The impact of cannula design on intra-nasal humidity, however, has not been investigated to date. OBJECTIVE: First, to develop a system, that samples air from the nasal cavity and analyzes the humidity of these samples. Second, to investigate nasal humidity during pre-nasal and intra-nasal oxygen application, with and without humidification. METHODS: We first developed and validated a sampling and analysis system to measure humidity from air samples. By means of this system we measured inspiratory air samples from 12 subjects who received nasal oxygen with an intra-nasal and pre-nasal cannula at different flows, with and without humidification. RESULTS: The sampling and analysis system showed good correlation to a standard hygrometer within the tested humidity range $(r=0.99, P<.001)$. In our subjects intranasal humidity dropped significantly, from $40.3 \pm 8.7 \%$ to $35.3 \pm 5.8 \%, 32 \pm 5.6 \%$, and $29.0 \pm 6.8 \%$ at flows of 1,2 , and $3 \mathrm{~L}$, respectively, when oxygen was given intra-nasally without humidification $(P=.001, P<.001$, and $P<.001$, respectively). We observed no significant change in airway humidity when oxygen was given pre-nasally without humidification. With the addition of humidification we observed no significant change in humidity at any flow, and independent of pre- or intranasal oxygen administration. CONCLUSIONS: Pre-nasal administration of dry oxygen achieves levels of intranasal humidity similar to those achieved by intranasal administration in combination with a bubble through humidifier. Pre-nasal oxygen simplifies application and may reduce therapy cost. Key words: nasal humidity; oxygen therapy; nasal oxygen cannula; nasal mucosa. [Respir Care 2013;58(8): 1323-1328. (c) 2013 Daedalus Enterprises]
\end{abstract}

\section{Introduction}

Oxygen administration via nasal cannula was established several decades ago. ${ }^{1}$ The use of oxygen increased re-

\footnotetext{
The authors are affiliated with the Department of Pulmonology, Intensive Care, and Sleep Medicine, Fachkrankenhaus Kloster Grafschaft, Schmallenberg, Germany.

The authors have disclosed no conflicts of interest.

Correspondence: Dominic Dellweg MD, Department of Pulmonology, Intensive Care, and Sleep Medicine, Fachkrankenhaus Kloster Grafschaft, Annostraße 1, 57392 Schmallenberg, Germany. E-mail: d.dellweg@fkkg.de.
}

DOI: $10.4187 /$ respcare.02215 markably after publication of 2 landmark studies in the 1980s, which have shown that long-term oxygen administration in COPD patients with hypoxemia results in a near doubling of life expectancy., ${ }^{2,3}$ For long-term oxygen therapy the usual prescribed flow is $2 \mathrm{~L} / \mathrm{min}$.

Several oxygen application systems have been marketed. Nasal cannulas are most frequently used, especially in Europe. Because all application systems deliver absolutely dry oxygen, humidification is recommended by some, ${ }^{4}$ but not all, guidelines. ${ }^{5,6}$ If humidification is used, the most widespread system is the bubble through humidifier.

The velocity of the oxygen flow streaming out of a nasal cannula with an inner diameter of $3 \mathrm{~mm}$ and a dose of $2 \mathrm{~L} / \mathrm{min}$ is $4.7 \mathrm{~m} / \mathrm{s} .^{7}$ Exposure to dry and undiluted oxygen at such a high flow velocity may cause mucosal dryness and irritation. Chronic exposure may cause local inflam- 
mation, bleeding of the mucosa, and possibly nasal-septal perforation..$^{8-11}$ On the other hand bubble through humidifiers may cause infections if the water is contaminated with bacteria. ${ }^{12-15}$ Additionally, the noise of the bubbles may be annoying and may disturb sleep.

Oxygen masks as well as pre-nasal cannula systems have outlets of greater cross sectional area and emit oxygen outside the nostrils. The pre-nasal cannula used in this investigation (Fig. 1) has outlets toward the nose and toward the mouth. This creates a little stable cloud of oxygen in front of the nose, which enables a sufficient oxygen flow into the lung not only during nose breathing but also during mouth breathing. ${ }^{7}$ Pre-nasal oxygen administration has been shown to be equally effective to oxygen administration via face mask in terms of oxygenation. ${ }^{7}$ Face mask administration, on the other hand, is at least as effective as intra-nasal oxygen administration. ${ }^{16}$

In theory, the dry oxygen can absorb humidity from the surrounding air along the way from the probe outlet to the nose orifice. This process could potentially make humidification unnecessary. To test this hypothesis we measured the humidity inside the nasal cavity with a pre- and intranasal cannula system, with and without humidification. First, however, we had to develop a system able to sample air from the nasal cavity and analyze the humidity of these samples.

\section{Methods}

\section{Ethics}

The protocol and equipment were approved by the ethics committees of the medical council and of the University of Muenster, Westfalen, Germany (2010-583-f-S). Informed consent was obtained from all subjects.

\section{Measurement System}

The modified Swan-Ganz catheter (TD 7F, Corodyn, Braun, Melsungen, Germany), originally used for measurement of vascular pressure and cardiac output, is shown

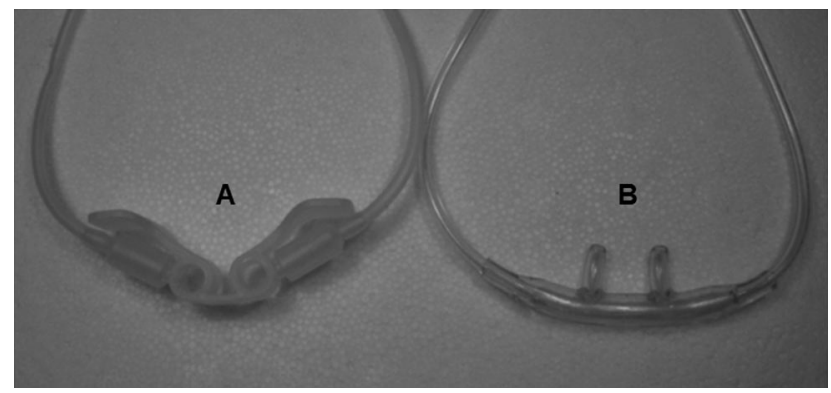

Fig. 1. Applicator systems. A: Oxynasor pre-nasal cannula. $\mathrm{B}$ : Covidien adult nasal cannula.

\section{QUICK LOOK}

\section{Current knowledge}

Low-flow oxygen is commonly delivered with an unheated bubble humidifier or no humidifier. Low-flow oxygen can dry the nasal mucosa and cause discomfort and increased nasal airway resistance.

\section{What this paper contributes to our knowledge}

Pre-nasal administration of dry oxygen had intranasal humidity similar to intranasal administration with bubble humidifier. Pre-nasal oxygen simplifies application and may reduce costs.

in Figure 2. The distal catheter end was cut off directly above the temperature sensor. This temperature sensor was used to measure air temperature during the sampling procedure, and 2-point calibration of the temperature sensor was performed prior to measurements. The catheter has a diameter of 7 French, and 4 channels. The first channel contains a wire that connects to the built-in temperature sensor on the catheter tip. The second channel was used to suck in air samples. The remaining 2 channels were used to slide in a Constatan heating wire that was bent to build a $180^{\circ} \mathrm{U}$-turn at the catheter tip. A small cage at the catheter tip prevents direct contact of the temperature probe and the nasal wall.

Because the humidity in the nasal cavity changes considerably within the breathing cycle, we had to measure chest excursions to ensure that air was sampled during inspiration. Thoracic movements were detected with a standard piezoelectric belt (Polymesam, MAP MedizinTechnologie/ResMed, Martinsried, Germany); the amplified signal was connected to a standard analog volt meter, to visualize the respiratory effort. The air sampling device was manually activated at onset of inspiration, and was programmed to operate for a duration of 0.8 seconds.

During this time the pump sucks in a volume of $12 \mathrm{~mL}$ from the tip of the catheter into the measuring chamber. The volume of the sampling channel of our catheter was determined to be $1 \mathrm{~mL}$, the content of the measurement chamber was determined to be $10 \mathrm{~mL}$. In order to analyze a representative sample, we analyzed air taken from the third of 3 consecutive suction maneuvers of 3 consecutive breaths. Air samples were kept in the measurement chamber until the hygrometer recorded stable measurement results, which was achieved within seconds. All experiments were carried out by the same scientist.

In our setting the temperature in the nostril (body temperature) was warmer than the surrounding room air. To avoid condensation of humidity in the catheter during sampling, the whole catheter was heated to approximately $45^{\circ} \mathrm{C}$. 


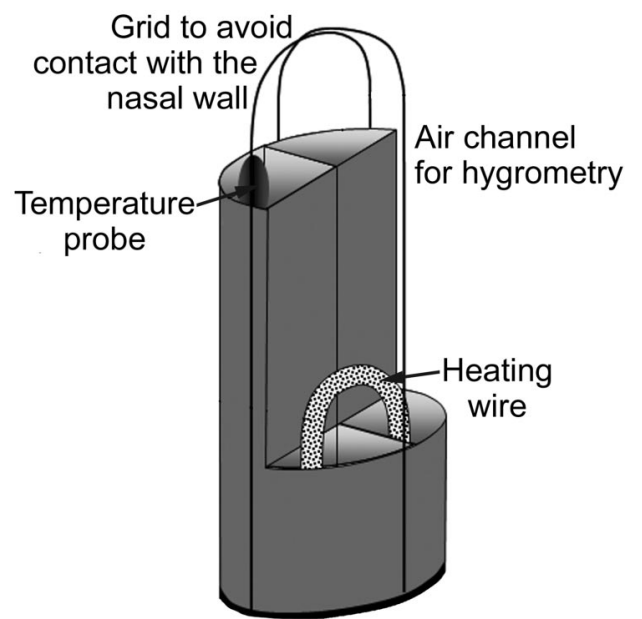

Fig. 2. Tip of the modified Swan-Ganz catheter. The first channel was used to take air samples. The second channel was used for temperature measurements at the sampling site. The 2 remaining channels contain a heating wire to keep the catheter temperature constant.

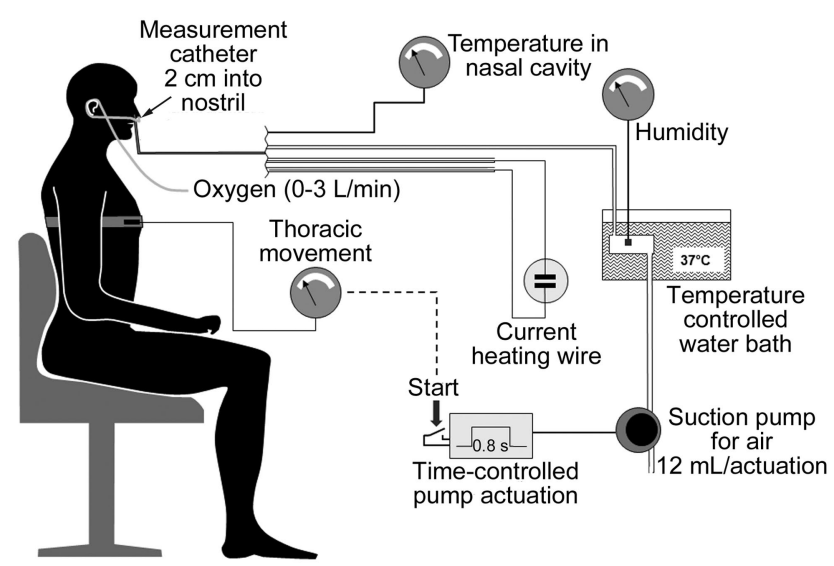

Fig. 3. Experimental setup. Inspiratory air samples were drawn inside the subject's nostril, and humidity was analyzed in a chamber that was kept constantly at $37^{\circ} \mathrm{C}$.

The humidity of the sampled volume was measured under steady conditions in a water bath at $37^{\circ} \mathrm{C}$ (Koettermann 3041, Koettermann, Haenigsen, Germany) with an industrial sensor (Hygrotest 6400, Testo, Lenzkirch, Germany). The entire measurement system is shown in Figure 3.

\section{Calibration of the Measurement System}

To validate our measurement device we performed comparative measurements with a standard industrial hygrometer (Testo 625, Testo, Lenzkirch, Germany). We modified humidity inside a container by addition of water-soaked cotton balls of different water content under room air conditions. Humidity was measured with a standard hygrom- eter inside the container, as well as from air samples taken by means of our newly developed sampling device.

\section{Comparison Study}

Figure 1 shows the tested cannula systems, a standard nasal cannula (Covidien nasal cannula adult REF 13300, Salter Labs, Arvin, California) and a pre-nasal cannula (Oxynasor HLM.27.001, Heinen \& Löwenstein, Bad Ems, Germany).

The measurements were done in a randomized order for either oxygen application system, as well as randomization of flow and whether humidification was added or not. All subjects inhaled oxygen flows of $0,1,2$, and $3 \mathrm{~L} / \mathrm{min}$, with and without a bubble through humidifier (Kendall Respiflo H, 500 mL, Covidien, Mansfield, Massachusetts) with either cannula system. The different runs were interrupted by a 5-min pause where subjects were breathing room air only in order to guarantee equal entry criteria.

During quiet nose breathing the sampling device was activated during 3 consecutive breaths, to assure complete wash-out of the measurement chamber (see Fig. 2). At the same time, the temperature in the nasal cavity was measured. We repeated every measurement 3 times and subsequently averaged the results. The humidity in the measurement chamber was converted to the temperature conditions measured in the nasal cavity according to the Magnus formula. ${ }^{17}$ The 12 subjects were recruited from the staff of different departments of our hospital.

\section{Statistics}

Data are expressed as mean and standard deviation. A $P$ of $<.05$ was considered significant. Differences were analyzed by 3 factor analysis of variance, evaluating the effect of flow, humidification, and device, after performing the Levene test to proof for homogeneity of the variances. Post hoc analysis was done by means of the Scheffé procedure. The Pearson correlation test was used within the validation study after normal distribution of data were confirmed by the Kolmogorov-Smirnov test. We used statistics software (SPSS 20, SPSS, Chicago, Illinois) for analysis.

\section{Results}

The results of the system calibration measurements showed excellent correlation between the standard hygrometer and our newly developed device, with a Pearson correlation coefficient of $0.99(P<.001)$.

The data obtained from the 12 subjects $(20-60 \mathrm{y}, 4 \mathrm{fe}-$ male) are shown in Figure 4. The average temperature inside the nasal cavity of our subjects during inspiration was determined to be $30.8 \pm 1.6^{\circ} \mathrm{C}$, and thus had in- 


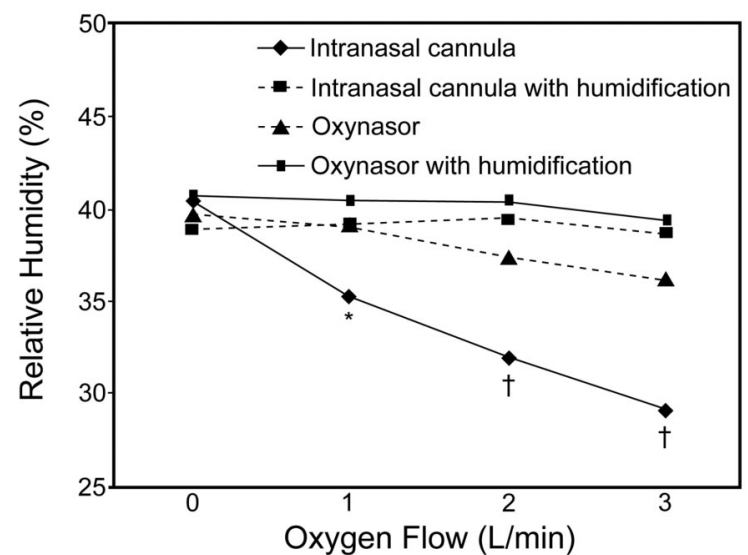

Fig. 4. Relative humidity at different oxygen flows with the different cannula systems. ${ }^{*} P=.001$ compared to zero flow. $\dagger P<.001$ compared to zero flow.

creased already above room temperature. With humidification no significant difference in relative humidity of the nasal cavity was observed, independent of the oxygen application device and oxygen flow.

When the standard nasal cannula was applied without humidification, we observed a relevant and significant drop in relative humidity of the nasal cavity from $40.3 \pm 8.7 \%$ to $35.3 \pm 5.8 \%, 32 \pm 5.6 \%$, and $29.0 \pm 6.8 \%$ for the flow $1 \mathrm{~L} / \mathrm{min}(P=.001), 2 \mathrm{~L} / \mathrm{min}(P<.001)$, and $3 \mathrm{~L} / \mathrm{min}$ $(P<.001)$, respectively (see Fig. 4$)$. When oxygen was given pre-nasally without humidification, relative nasal humidity was not significantly affected at any flow (see Fig. 4).

\section{Discussion}

In this study we have shown, using a newly developed system for measuring relative humidity, that relative humidity in the nasal cavity drops significantly, even at low flow of intra-nasally administered dry oxygen. At a flow of $3 \mathrm{~L} / \mathrm{min}$, humidity had already dropped by one third. Given as long-term treatment, this might result in nasal discomfort due to dryness and irritation, and could result in serious complication as described previously. ${ }^{8-11}$

Airway humidity levels during administration of dry oxygen given by means of the pre-nasal cannula were similar to those achieved with intranasal administration in combination with a bubble through humidifier, and makes the use of this humidification device unnecessary. This observation leads to the assumption that the exchange of humidity from the ambient air to the dried oxygen occurs very rapidly and before the air enters the nasal cavity. Our results are in line with previous data that compared nasal cannulas to a system that delivers oxygen via a larger opening in front of the mouth. ${ }^{18}$
In general, humidity describes the amount of water vapor in gases. Humidity can be given as the absolute mass of water per unit of volume, which is the absolute humidity, or as a fraction of full saturation under constant conditions of volume, temperature, and pressure, which is called relative humidity. Usual indoor air at atmospheric pressure and at a temperature of $20^{\circ} \mathrm{C}$ has a relative humidity of about $55-60 \% .{ }^{19}$ During inspiration the temperature of the inspired gas will increase and approach body temperature within the distal airways. The onset of this effect can be seen in our experiments, since the nasal temperature was already $30.8^{\circ} \mathrm{C}$ and thus above room air temperature, while intranasal relative humidity was determined not to be higher than $42 \%$, even at zero flow (see Fig. 4). Given the same absolute humidity, an increase in temperature will result in a drop in relative humidity, a phenomenon we observed, as nasal temperature was above room temperature. Thus, the increase in temperature appears to occur faster than the rise in relative humidity within the natural airways.

During the further passage toward the alveoli, the inspired gas will not only be heated to body temperature but will also increase relative humidity up to $100 \%$ by water uptake from the mucosal surface of the airways. ${ }^{19,20}$

Our experiments provide information only about the impact of oxygen humidification on intranasal humidity. Whether humidity in the more distal airways is affected as well, or if water uptake from the mucosal airways during inspiration might compensate this deficit, was beyond the scope of our investigation. However, decreased humidity in the nasal cavity by application of dry oxygen, as seen in our experiment, increases the capacity and propensity for water uptake from the mucosal surface.

Whether our data are clinically relevant cannot be surely answered from this examination, because the application time was short and we examined healthy subjects. The discussion about humidification of oxygen during longterm oxygen therapy is controversial. Campbell et $\mathrm{al}^{21}$ did not see differences in side effects between humidified and dry oxygen given intra-nasally. In his study, however, he examined postoperative patients after cardiac surgery who received oxygen for an average of only less than 3 days. Patients received either dry or humidified oxygen, and thus were not able to compare both treatment modalities. Their complaints were categorized into dry throat, dry nose, headache, chest discomfort, and other complaints, overall symptoms that are quite common after cardiac surgery under general anesthesia. Some guidelines go along with Campbell's results and do not recommend the routine use of humidification systems.5,6 Andres et al conducted a similar study using a cross-over design. ${ }^{22} \mathrm{He}$ found significant differences in symptom scores, but not in the incidence of nose bleeds. The latter, however, was 
unlikely to happen, given the short treatment time of only 3 days.

Miyamoto and co-workers confirmed these results in a carefully designed study with a comparison of humidified versus dry oxygen, in a cross-over design, at flows between 1 and $5 \mathrm{~L} / \mathrm{min} .{ }^{10}$ Although the application time was very short (1 min), nasal discomfort was recognized by healthy subjects at any tested flow, while subjects with pulmonary disorders, who were likely to be used to oxygen administration, perceived nasal discomfort only at flows of $>2 \mathrm{~L} / \mathrm{min}$. Miyamoto's findings raise the question if the subjective perception of nasal discomfort might by subjected to habituation. With a flow greater than $5 \mathrm{~L} /$ min, even bubble through humidifiers appear to have a ceiling effect, since they were perceived to be inferior to heated humidifying systems. ${ }^{11}$ Air from heated humidifiers is usually warmer than ambient air and thus can accumulate and deliver more humidity. A possible drawback, however, might be the development of condensate due to the cooling down within the tubing system.

For optimal treatment and, more importantly, to prevent complications, one should consider the oxygen application device, the type of humidification, and treatment duration. Humidifying systems carry the potential risk of contamination. Previous studies did not find differences in bacterial contamination rates of prefilled disposable versus multiuse oxygen humidifiers. ${ }^{13}$ No contamination differences were found when humidifiers were filled with sterile or tap water. ${ }^{23}$ While some authors found no or negligible contamination rates ${ }^{13,24,25}$ and proposed even multipatient use of humidifiers, ${ }^{25}$ other investigators found relevant contamination rates of potentially pathogenic organisms. 12,23,26 There are no epidemiologic studies that translate these finding into clinical outcomes such as infection rate, hospital stay, or even death, although there is evidence that humidifier contamination is a risk for infection. ${ }^{14,15}$

For this study we developed a measurement device that can take air samples through a catheter and determine sample-air humidity under constant conditions. With knowledge of the local temperature at the sample site, which is also being determined by means of the built-in temperature sensor, one can calculate the relative humidity at the sampling site. We used this device within the nasal cavity; however, it appears feasible to apply this technique to more distal areas of the human respiratory tract for appropriate research questions.

\section{Limitations}

Calibration measurements were done at room temperature, while subject measurements were conducted at $37^{\circ} \mathrm{C}$. The hygrometer we built into our measurement system, however, has a validated measurement range from $-199.9^{\circ} \mathrm{C}$ to $199.9^{\circ} \mathrm{C}$, and thus should provide reliable data for our experiments. We did not assess nasal discomfort in our subjects, and, due to the short period of the exposure to the different experimental settings, our data cannot assess any clinical outcome. We limited our study to one type of humidifier and cannot exclude the possibility that different modes of humidification could have influenced our measurements differently.

\section{Conclusions}

We introduced a helpful and valid tool to measure relative humidity in air-filled body cavities such as the nasal cavity. Nasal humidity drops significantly when dry oxygen is given intra-nasally, even at low flow. Pre-nasal application of dry oxygen with low flow velocity at the outlet does not impact nasal humidity, at least up to a flow of $3 \mathrm{~L} / \mathrm{min}$, and thus obviates the need for additional humidification. With this approach, oxygen therapy might become less cumbersome, cheaper, and more hygienic.

\section{REFERENCES}

1. Marriott HL. Oxygen administration by nasal catheter. BMJ 1936; 1(3916):154-157.

2. Nocturnal Oxygen Therapy Trial Group. Continuous or nocturnal oxygen therapy in hypoxemic chronic obstructive lung disease: a clinical trial. Ann Intern Med 1980;93(3):391-398.

3. Long term domiciliary oxygen therapy in chronic hypoxic cor pulmonale complicating chronic bronchitis and emphysema. Report of the Medical Research Council Working Party. Lancet 1981;1(8222): 681-686.

4. Magnussen H, Goeckenjan G, Kohler D, Matthys H, Morr H, Worth $\mathrm{H}$, et al. [Guidelines to long-term oxygen therapy]. Pneumologie 2001;55(10):454-464. Article in German.

5. O'Driscoll BR, Howard LS, Davison AG; British Thoracic Society. BTS guideline for emergency oxygen use in adult patients. Thorax 2008;63(Suppl 6):vi1-68. Erratum in: Thorax 2009;64(1):91.

6. American Thoracic Society. Standards for the diagnosis and care of patients with chronic obstructive pulmonary disease. Am J Respir Crit Care Med 1995;152(5 Pt 2):S77-S121.

7. Köhler D, Knoch M, Sommerfeld C, Müller H. A new oxygen applicator for simultaneous mouth and nose breathing. Chest 1993; 103(4):1157-1160.

8. Kelly MG, McGarvey LP, Heaney LG, Elborn JS. Nasal septal perforation and oxygen cannulae. Hosp Med 2001;62(4):248.

9. Wu K, Ahmed A, Woolford TJ. Treatment of home oxygen induced rhinitis: an unusual use for a nasal obturator. Rhinology 2004;42(4): 244-245.

10. Miyamoto K, Nishimura M. Nasal dryness discomfort in individuals receiving dry oxygen via nasal cannula. Respir Care 2008;53(4): 503-504.

11. Chanques G, Constantin JM, Sauter M, Jung B, Sebbane M, Verzilli $\mathrm{D}$, et al. Discomfort associated with underhumidified high-flow oxygen therapy in critically ill patients. Intensive Care Med 2009; 35(6):996-1003.

12. Pendleton N, Cheesbrough JS, Walshaw MJ, Hind CR. Bacterial colonisation of humidifier attachments on oxygen concentrators prescribed for long term oxygen therapy: a district review. Thorax 1991; 46(4):257-258. 


\section{HUMIDIFICATION OF INSPIRED OXYGEN}

13. Yamashita K, Nishiyama T, Yokoyama T, Abe H, Manabe M. A comparison of the rate of bacterial contamination for prefilled disposable and reusable oxygen humidifiers. J Crit Care 2005;20(2): 172-175.

14. Bou R, Ramos P. Outbreak of nosocomial Legionnaires' disease caused by a contaminated oxygen humidifier. J Hosp Infect 2009; 71(4):381-383.

15. Harris AA, Goodman L, Levin S. Community-acquired Pseudomonas aeruginosa pneumonia associated with the use of a home humidifier. West J Med 1984;141(4):521-523.

16. Catterall M, Kazantzis G, Hodges M. The performance of nasal catheters and a face mask in oxygen therapy. Lancet 1967;1(7487): 415-417.

17. Lawrence MG. The relationship between relative humidity and the dew point temperature in moist air: a simple conversion and applications. Bull Am Meteorol Soc 2005;86:225-233.

18. Paul J, Otvos T. Comparison of nasal cannulas and the OxyArm in patients requiring chronic domiciliary oxygen therapy. Can Respir J 2006;13(8):421-426.

19. Gross JL, Park GR. Humidification of inspired gases during mechanical ventilation. Minerva Anestesiol 2012;78(4):496-502.
20. Dery R. Humidity in anaesthesiology. IV. Determination of the alveolar humidity and temperature in the dog. Can Anaest Soc J 1971; 18(2):145-151.

21. Campbell EJ, Baker MD, Crites-Silver P. Subjective effects of humidification of oxygen for delivery by nasal cannula. A prospective study. Chest 1988;93(2):289-293.

22. Andres D, Thurston N, Brant R, Flemons W, Fofonoff D, Ruttinmann A, et al. Randomized double-blind trial of the effects of humidified compared with nonhumidified low flow oxygen therapy on the symptoms of patients. Can Respir J 1997;4(2):76-80.

23. Cahill CK, Heath J. Sterile water used for humidification in lowflow oxygen therapy: it is necessary? Am J Infect Control 1990; 18(1):13-17.

24. Seigel D, Romo B. Extended use of prefilled humidifier reservoirs and the likelihood of contamination. Respir Care 1990;35(8):806.

25. Golar SD, Sutherland LL, Ford GT. Multipatient use of prefilled disposable oxygen humidifiers for up to 30 days: patient safety and cost analysis. Respir Care 1993;38(4):343-347.

26. Cameron JL, Reese WA, Tayal VS, Clark RF, Kelso D, Gonzalez $\mathrm{ER}$, et al. Bacterial contamination of ambulance oxygen humidifier water reservoirs: a potential source of pulmonary infection. Ann Emerg Med 1986;15(11):1300-1302. 